\title{
Carnets
}

Revue électronique d'études françaises de l'APEF

Deuxième série - 21 | 2021

Arts du vin

\section{La Poétique de Jean-Charles Chapuzet}

Entre roman œnotouristique et polar viticole

José Domingues de Almeida

\section{CpenEdition}

Journals

Édition électronique

URL : https://journals.openedition.org/carnets/12654

DOI : $10.4000 /$ carnets. 12654

ISSN : 1646-7698

Éditeur

APEF

Référence électronique

José Domingues de Almeida, "La Poétique de Jean-Charles Chapuzet », Carnets [En ligne], Deuxième série - 21 | 2021, mis en ligne le 31 mai 2021, consulté le 01 juin 2021. URL : http://

journals.openedition.org/carnets/12654; DOI : https://doi.org/10.4000/carnets.12654

Ce document a été généré automatiquement le 1 juin 2021

\section{(c) (†) \&)}

Carnets est mis à disposition selon les termes de la licence Creative Commons - Atribution - Pas d'utilisation commerciale 4.0 International. 


\section{La Poétique de Jean-Charles Chapuzet}

Entre roman œnotouristique et polar viticole

José Domingues de Almeida

\section{NOTE DE L'AUTEUR}

Cet article est financé par les fonds FEDER du Programme d'Exploitation des Facteurs de Compétitivité - COMPETE (POCI-01-0145-FEDER-007339) et par les fonds nationaux de la FCT - Fondation pour la science et la technologie, dans le cadre du projet stratégique «UID/ELT/00500/2013».

1 Le journaliste, historien, essayiste, reporter, romancier, mais aussi œnologue français, Jean-Charles Chapuzet, né en 1976 en Charente-Maritime - terre de vignobles - s'est surtout signalé médiatiquement par son intérêt pour les personnages atypiques, au destin hors du commun. Il suffit pour s'en convaincre d'évoquer son sujet de thèse de doctorat en sciences politiques sur la personnalité du général Boulanger ${ }^{1}$, ou encore l'intérêt qu'il porte à la carrière du compositeur-interprète Hubert Félix Thiéfaine. Mais on pourrait aussi dégager son livre-enquête sur le personnage charentais excentrique, tout aussi original, de «L'homme à la soucoupe ", l'insolite Jean-Claude Ladrat dans Mauvais plan sur la comète (2018).

2 Cependant, c'est surtout sa passion du vin, ainsi que son attachement affectif aux terroirs, alliés à son goût du polar qui retiendront notre attention dans cette étude. En effet, Jean-Charles Chapuzet accumule des collaborations et des études sur les vignobles: Terre de vins (2011), Cuvée Sismique (2014) ou Bordeaux 1855 : Les grands crus classés (2015) chez l'éditeur régional grenoblois Glénat, au couvent Sainte-Cécile, chez qui il publiera plusieurs polars ancrés dans ces contrées viticoles qu'il connaît si bien. Il publiera aussi chez Féret, à Bordeaux.

3 Nous proposons une lecture de deux romans publiés chez ces deux éditeurs, deux récits procurant respectivement une approche de la fiction que l'on pourrait désigner de 
«œnotouristique » - Verticale (2009)2 , ainsi qu'un polar dans lequel l'enquête porte sur les cavistes et les vignerons - À l'aveugle (2013) ${ }^{3}$. Ces deux textes fictionnels ont ceci en commun qu'ils plantent d'un point de vue thématique un décor, et invoquent un imaginaire qui exalte le vin et le vignoble comme éléments mythiques et fusionnels du terroir. Par ailleurs, ils convoquent, d'un point de vue critique, une approche écocritique, voire configurent ce que d'aucuns ont, à propos de Chapuzet, baptisé de fiction «œnotouristique $»^{4}$, mélange de petites histoires avec la (re)découverte des terroirs viticoles.

4 En effet, dans le roman Verticale, Bertrand, un jeune maître de conférences bourguignon - au profil en tout ressemblant à celui de l'auteur puisque «les titres donnaient un indice sur ses sujets de prédilections : beaucoup de polars anglo-saxons, de bandes dessinées et de livres d'histoire touchant de près ou de loin au vin » $(V: 11)$ et avec un sujet de thèse de doctorat portant sur la « 'Violence et politique en terre vigneronne'« (ibidem) - et son amie, Audrey, étudiante en botanique et éprise de la passion du vin, décident de partir en Charente Maritime en $2 \mathrm{CV}$ pour le goût de l'évasion pure : «Restait à traverser la France, la mèche d'Audrey au vent » $(V: 17)$. Ils désignent comme destination les Côtes de Bourg dans le Sud-Ouest. Si, apparemment, les deux personnages sont partis pour s'amuser et visiter cette région viticole qui regorge de trésors architecturaux, de célébrités, de légendes (celle de la Vierge manchote, notamment ( $V: 91)$ et d'Histoire, d'autres péripéties et intentions viendront complexifier et épaissir le récit.

5 Les dialogues, l'observation et la description des paysages sont d'abord l'occasion d'une évocation en connaisseur d'épisodes marquants du passé des contrées traversées. Bertrand s'avère être ce guide aussi bien œnotouristique qu'historique: "Bertrand contait à son amie les origines de Bourg-sur-Gironde, site prisé par les Gaulois suivis par les Romains pour sa position stratégique évidente » $(V: 19)$, ou encore :

Ce pays devait beaucoup aux communautés religieuses. Durant les premiers siècles de notre ère. La conquête chrétienne accompagna l'importante consécration du vignoble. La foi n'allait pas sans vin de messe. Greffés dans un premier temps aux villages, les vignobles s'étaient ensuite étendus dans les terres, au point de devenir le pouvoir économique de la région $(V: 100)$.

6 Nous ne sommes certes pas loin ici de la «pensée-paysage » et du regain d'intérêt fictionnel pour le paysage chers à Michel Collot (2011 : 11), à savoir « (...) des pratiques paysagères, qui récusent les méfaits du modernisme et réinventent sous des formes et avec des moyens nouveaux l'ancienne alliance entre l'être humain et son environnement» (idem: 13), et qu'il complexifie ainsi : "Le paysage apparaît ainsi comme une manifestation exemplaire de multidimensionalité des phénomènes humains et sociaux, de l'interdépendance du temps et de l'espace, et de l'interaction de la nature et de la culture, de l'économique et du symbolique, de l'individu et de la société » (idem: 11). Et Collot de dégager une phénoménologie paysagère qui engagerait :

(...) l'interaction entre un site, sa perception et sa représentation. (...) C'est le regard qui transforme le site en paysage et qui rend possible son 'artialisation'. (...) Tel qu'il se manifeste dans l'expérience du paysage, notre rapport sensible au monde n'est pas celui d'un sujet posé en face d'un objet, mais celui d'une rencontre et d'une interaction permanentes entre le dedans et le dehors, le moi et l'autre (idem: 18-28). 
7 On pourrait légitimement considérer la fiction œnotouristique de Jean-Charles Chapuzet une dérivation thématique, géographique et paysagère de cette approche. En effet, l'évasion de Bertrand et d'Audrey - qui bien évidemment auront une affaire, paysage et vin aidant - induit l'interaction avec le terroir, fait émerger cette osmose entre paysage, fiction et sujet : «C'était comme si rien n'avait changé depuis des siècles. Le paysage vivait au rythme de la taille, au gré des récoltes. C'était toujours un peu de souffrance et de bonheur qui se lisait dans la beauté de la terre » $(V: 30)$; «C'était dans ce décor intemporel si particulier que Bertrand et Audrey partageaient un moment » $(V: 32)$.

8 Par ailleurs, deux éléments narratifs viennent - nous le disions plus haut - donner de l'épaisseur au récit, sans quoi il en resterait sur le registre purement descriptif et touristique. D'une part, un meurtre dont nos deux amis et amants sont, sinon les témoins, du moins les acteurs passifs et involontaires, alors qu'ils faisaient l'amour sur un site viticole :

Soudain, un bruit de feuilles se fit plus insistant. Une branche claqua. Audrey sursauta, son cœur se mit à battre la chamade. Bertrand s'arrêta de bouger. Il pensa à un sanglier ou à une autre bête quelconque. Mais le souffle qu'ils entendirent fut clairement celui d'un homme. Bertrand posa la main sur la bouche d'Audrey qui s'affolait à se revêtir $(V: 42-43)$.

9 D'autre part, dans cette échappée en territoire viticole, Bertrand cache à Audrey son intention de retrouver son père génétique, une quête personnelle qui bute sur l'accueil distant et décevant de cet homme :

Bertrand ne l'écoutait pas. Il remarqua une sorte de rire inquiet accroché aux lèvres de cet homme. Il en était sûr. C'était son père. Il avait un certain attendrissement. Il crut, l'espace d'une seconde, à un conte de fée. La rencontre (...). Et puis (...) [il] passa à un sentiment de déception. Ce n'était pas celui qu'il s'était imaginé, enfant, avant de s'endormir, la tête pleine d'interrogations ( $V: 107)$.

Mais ce rendez-vous manqué assume un autre cas de figure quand, après enquête, on découvre que l'auteur du meurtre n'est autre que le demi-frère de Bertrand. Dès lors, le roman prend pour prétexte un fait divers colporté par la presse locale et sur lequel les deux amants devront témoigner : “'Un meurtre à Marmisson ?'. L'article était annoncé en Une mais le titre avait échappé à Bertrand, loin de penser que cette information pouvait le concerner» $(\mathrm{V}: 58)$. Le fait divers criminel - que Jean-Charles Chapuzet affectionne - s'avère le moteur de la progression narrative. En effet, "le fait divers transgresse, [et] porte atteinte au déroulement normal ou conventionnel des choses » (Dubied \& Lits, 1999: 53), ce que Roland Barthes nommait la «mise en scène de l'extraordinaire et du surprenant » (1964:188-197) ou, pour reprendre Barillaud et al., « l'intuition d'une incompatibilité » (1986:57), qui ouvre la voie à son esthétisation par ses points de convergence avec la construction romanesque, notamment pour ce qui est d'un certain éloignement du normal (1986: 212-215).

11 Or, ici, l'esthétisation passe par le vin, et il n'en faut pour preuve que le titre du roman. En effet, les œnologues nous diront qu'une dégustation "verticale » se réfère à un voyage au fil des millésimes. La "quête inlassable de racines" ( $V: 108)$ par Bertrand s'apparente explicitement à cette méthode œnologique évoquée au cours d'une dégustation dans un chai: «La verticale allait de 1985 à 2003 " $V:$ 126). En dénouement, Bertrand justifiera la motivation de sa randonnée charentaise : «- Dans le vin, tu me disais que le hasard n'existe pas. Dans notre histoire non plus. En réalité... mon père habite ici...» $(V: 129)$, pour avouer plus loin à Audrey que «C'est mon 
histoire, mon passé, ma verticale à moi, si tu préfères... » (ibidem), et le narrateur de conclure par une sorte de "tout est bien qui finit bien" un peu morne: "Bertrand connaissait enfin ses origines, sa verticale avait un sens. Voilà tout » $(V: 149)$.

Dans ce roman, le vin est forcément un motif majeur qui permet de faire fusionner tous les autres éléments. Le monde vinicole sans cesse convoqué garantit une approche mythique de ce breuvage, ce qui cautionne l'épithète « œnotouristique » que la critique a appliquée à ce récit : alliance du vin, du terroir et d'envie de découverte : «Dans ce tableau, tout était une question de lien à la terre » $(V: 7)$. Le couple de Bourguignons se reconnaît dans cet attachement irrésistible au vin: «Elle [Audrey] partageait avec Bertrand l'amour du vin, non pas pour l'histoire mais pour le goût et, accessoirement, l'ivresse » $(V: 12)$, ou encore : « Le vin, le vin..., il n'y a que le vin dans la vie (...)» (V : 20).

Dans À l'aveugle (2013) - titre à nouveau tiré du lexique œnologique, et qui définit la dégustation d'un vin sans en connaître à l'avance l'origine ${ }^{5}$ - Jean-Charles Chapuzet réinvestit le vignoble et s'aventure dans le polar vigneron qu'il affectionne, alliant intrigue, terroir et univers viticole dans le Rhône, cette fois. En effet, pendant la noce d'un couple de jeunes vignerons (Sarah-Jeanne et Pascal) à Signargues, leur récolte de grenache est volée, vendangée clandestinement. Après avoir fait de la prison, Hippolyte Lapaque est devenu œnologue et assure la rubrique œnologique d'un journal local: «(...) journaliste irascible, au verbe irrégulier, vivant, dormant, ripaillant au village de Saint-Pantaléon-les-Vignes » ( $A: 15)$, ce qui l'oblige à faire ce qu'il aime le plus : goûter de bons vins: "goûter des blancs" $(A: 12)$. Or, un ballon avec un papier attaché provenant tout droit de la fête de mariage du couple de viticulteurs atterrit dans le jardin de l'œnologue et finit par mettre le chroniqueur sur la piste de Signargues et de son cru.

Entre-temps, le spécialiste de vins visite plusieurs chais, y déguste et évalue de grands crus, ce qui permet au narrateur de nous introduire dans les rituels et le savoir vinicoles, et de nous faire communier avec l'alchimie du vin :

Regarde ces terroirs de fou. On n'est pas loin d'Estézargues et de ses galets roulés, que le Rhône a oubliés quand il recouvrait la région. Ils font des vins incroyables. De la syrah, du grenache, du cinsault... C'est réglisse, sanguin, racé, camphré, y a du cassis, une belle acidité, de l'équilibre - et ça vaut des broutilles : cinq euros! ( $A$ : 11) ;

ou encore :

Les syrahs, ça m'excite, commenta-t-il. Elles ont un côté sage, timide, saintenitouche, et puis elles se lâchent, elles tapissent la bouche de fruits rouges, elles sont végétales, coquines, réglissées, tout en soie, faut toujours se méfier du vin qui dort... (A:24).

Ceci dit, À l'aveugle mobilise les moteurs essentiels minima du roman policier qu'il fait dériver vers l'univers œnologique donnant à lire un sous-genre improbable : le « polar vigneron ${ }^{6}$. Rappelons l'acception canonique du roman policier forgée notamment par Daniel Fondanèche, lequel met en avant le rôle du délit comme embrayeur narratif :

Le roman policier est donc, semble-t-il, la trace romanesque d'une quête ayant pour but de rétablir un équilibre qui a été rompu après une transgression sociale. C'est la remise en ordre stable d'un état social qui, pendant un temps, a été perturbé. Le début de cette normalisation est confié à un individu (policier, enquêteur, justicier), avant que la fin en soit confiée au fatum ou à l'institution judiciaire (Fondanèche, $2000: 4)$. 
ou encore :

Le roman policier obéit à des lois propres, à un fonctionnement interne complexe du récit, qui a beaucoup évolué depuis la création du genre. Le détective amateur et l'aventurier ont cédé le pas au policier, puis au " privé » (private eye), qui enquêtent avec des méthodes très variées afin d'identifier des coupables de nature très différente (idem : 3 ).

Dans le cas spécifique de À l'aveugle, nous avons affaire à un "récit d'énigme " (Lits, 1989 : 30-33) qui s'inscrit dans une « ouverture vers d'autres catégories » (idem : 35-36), notamment celle évoquée et mise en fiction par Jean-Pierre Alaux, qui fait de l'œnologue un enquêteur : «Où fouiner ? L'enquête était au point mort » $(A: 53)^{7}$. En effet, le récit va introduisant des indices et des suspects (Lits, 1989: 157-167) du détournement criminel de vendange. Lapaque pressent « le règlement de comptes » ( $A$ : 32) et croise, sans s'en rendre compte, un des complices du vol: «En reprenant un gorgeon, Lapaque se demanda encore quel était ce 'nez-de-bœuf' qu'il avait croisé au bistrot de Sérignan-le-Comtat... Une vieille connaissance ? Il le connaissait, bon Dieu !» $(A: 25)$.

Le flair enquêteur de l'œnologue le met "à l'aveugle », comme on goûte un bon vin, sur la piste de «notables du vin» $(A: 53)$ et de leur rituel du poker au sein d'une académie œnologique. À l'aveugle, Lapaque reconnaît le vin servi à table qui trahit le vol de Signargues, et dénonce les coupables, dont un complice, Pascal, le mari de SarahJeanne : «Pascal a dû monter le coup pour toucher les assurances et s'assurer la vente des bouteilles au black, chez Latienne, à Avignon et peut-être ailleurs... » $(A: 111)$.

Entre-temps l'univers du vin fournit ici une riche et constante allégorie pour toute l'activité humaine, amoureuse notamment: "Comme un œnologue qui goûterait du vin, Lapaque cherchait les défauts chez Sarah-Jeanne. En vain» (A: 30). Gaston Bachelard ne plaçait-il pas déjà la vigne sous le régime féminin du repos, du soin (care), et n'imaginait-il pas le vin comme «(...) un archétype substantiel du monde de la matière. Il peut être grand ou petit, gros ou délicat, fort ou léger, mais il est toujours pur. (1948 : 331) », foncièrement incompatible avec l'eau (idem : 326) ?

Le vin sauve le taulard du désespoir : «Dans mon malheur, j'ai découvert le vin » (A : 75). Lapaque est entré dans le monde vinicole comme on se convertit en prison, initié par un récidiviste : « Et là, il m'a parlé du vin, c'était de la poésie. Je n'y connaissais rien mais j'avais l'impression d'être dans les vignobles, j'étais en liberté avec lui, je buvais ses paroles... (...)» (A: 76). Dans cette approche véritablement alchimique, le vin fusionne avec la nature et la culture : «Le vin me serait resté étranger... Ensuite, j'ai pris goût à toute sorte de littérature, à la culture (...)» (A:76). Les rituels vinicoles, notamment la dégustation, acquièrent un statut quasiment sacramentel et liturgique : «Hippolyte fit tourner le vin rouge dans le verre (...). Un œil plus haut que l'autre, il jaugea la limpidité du contenu comme si l'avenir du monde en dépendait » $(A: 23)$.

En fait, dans ces deux récits, nous avons affaire à des personnages principaux fous amoureux du vin, dont les pratiques et le lexique technique œnologiques rejoignent l'approche passionnelle qu'en fait Bernard Pivot dans le Dictionnaire amoureux $d u$ vin (Pivot, 2006). Pour Hippolyte Lapaque, le vin est un destin auquel on ne peut échapper : «Un domaine : j'y sens du vin. Quand le ciel vous appelle... » (A:15). Ce n'est certes pas un simple loisir latéral, mais de l'ordre de la fusion, de la communion et de la transformation. C'est de l'attente, de la lenteur... Il renvoie 'radicalement' au terroir et à l'homme, alors que le vignoble engage un agencement poétique de l'espace, interpelle 
une géopoétique. C'est l'ici et l'au-delà, le milieu naturel, physique et aussi une singulière pratique, l'assurance du local et l'appel de l'universel ${ }^{8}$. Il réconcilie l'homme et le territoire, le sujet et le lieu, pour reprendre Bertrand Westphal dans une approche fictionnelle du lieu, en tant qu'espace humanisé, espace transformé en lieu (Westphal, $2007: 15)$ : « La vue d'un être humain dans cette nature belle et hostile lui fit du bien. La solitude rongeait les paysages » $(A: 66-67)$.

\section{BIBLIOGRAPHIE}

BACHELARD, Gaston (1948). La terre et les rêveries du repos. Paris : José Corti.

BARILLAUD, M.C, BIÈQUE, J. \& DAHLET, P. (1986). Un chien peut en écraser un autre. Le fait divers : aspects théoriques, pédagogiques, documentaires. Paris : CIEP/BELC.

BARTHES, Roland (1964). « Structure du fait divers », in Essais critiques. Paris : Seuil, pp. 188-198.

CHAPUZET Jean-Charles (2009). Verticale. Bordeaux : Féret.

CHAPUZET Jean-Charles (2011). Terre de vins. Grenoble : Grénat.

CHAPUZET Jean-Charles (2013). À l'aveugle. Grenoble : Grénat.

CHAPUZET Jean-Charles (2014). Cuvée sismique. Grenoble : Grénat.

CHAPUZET Jean-Charles (2014). Cuvée sismique. Grenoble : Grénat.

CHAPUZET Jean-Charles (2015). Bordeaux 1855 : Les grands crus classés. Grenoble : Glénat.

CHAPUZET Jean-Charles (2018). Mauvais Plan sur la comète : L'étrange odyssée d'un Haut Saintongeais. Montreuil : Éditions Marchialy.

Collot, Michel (2011). La pensée-paysage : philosophie, arts, littérature. Paris : Actes Sud.

DUBIED, Annik \& LITS, Marc (1999). Le fait divers. Paris: P.U.F., coll. « Que sais-je? ».

FONDANÈCHE, Daniel (2000). Le roman policier. Paris : Ellipses.

LITS, Marc (1989). Pour lire le roman policier. Paris / Gembloux : De Boeck-Duculot.

PIVOT, Bernard (2006). Dictionnaire amoureux du vin. Paris : Plon.

WESTPHAL, Bertrand (2007). La Géocritique. Réel, fiction, espace. Paris : Minuit.

\section{Sitographie :}

http://www.aquitaineonline.com/culture-livres-musique/livres-cursives/romanoenotouristique-cotes-de-bourg-jean-charles-chapuzet-09050401.html [disponible le 31/03/2020].

http://cote-du-rhone-news.over-blog.com/a-l-aveugle-polar-vigneron-de-jean-charles-chapuzet [disponible le 31/03/2020].

https://actu.fr/occitanie/toulouse_31555/le-polar-se-met-au-vin-et-alaux_3479228.html [disponible le 31/03/2020]. 
https://www.uc.pt/fluc/apef/act/pdf/100 [disponible le 31/03/2020].

\section{NOTES}

1. Qui fut, entre autres, ministre de la guerre à la fin du $\mathrm{XIX}^{\mathrm{e}}$ siècle, témoin d'événements bouleversants pour la France de l'époque, et eut une existence "romanesque » jusques et y compris dans son suicide.

2. Désormais $V$.

3. Désormais $A$.

4. Voir < http://www.aquitaineonline.com/culture-livres-musique/livres-cursives/romanoenotouristique-cotes-de-bourg-jean-charles-chapuzet-09050401.html>

5. «Il servit ses cuvées à l'aveugle aux côtés d'autres vins qui n'étaient pas de la région. 'La seule façon d'avoir une vue d'ensemble, c'est de déguster à l'aveugle', arguait-il » (A:52, voir aussi 46, 87).

6. Voir < http://cote-du-rhone-news.over-blog.com/a-l-aveugle-polar-vigneron-de-jean-charleschapuzet>

7. <https://actu.fr/occitanie/toulouse_31555/le-polar-se-met-au-vin-et-alaux_3479228.html>

8. Voir https://www.uc.pt/fluc/apef/act/pdf/100

\section{RÉSUMÉS}

Cet article propose une lecture axée sur la prégnance du vin et du monde rural et viticole dans deux romans de l'historien, journaliste et écrivain français contemporain, Jean-Charles Chapuzet : Verticale (2009) et À l'aveugle (2013). En effet, ces deux récits procurent respectivement une approche de la fiction que l'on pourrait désigner de « œnotouristique », ainsi qu'un polar qui enquête cavistes et vignerons. Ce faisant, ces deux textes - qui appellent, entre autres, une lecture écocritique - plantent un décor, et invoquent un imaginaire, qui exaltent le vin comme élément mythique et fusionnel du terroir.

This paper offers a reading focused on the importance of wine and the rural world in two novels by the historian, journalist and contemporary French writer, Jean-Charles Chapuzet: Verticale (2009) and À l'Aveugle (2013). Indeed, these two novels respectively provide an approach to fiction that could be called "wine tourism", as well as a thriller that investigates wine merchants and winegrowers. In doing so, these two texts - which call for, among other things, an ecocritic reading - set the scene, and invoke an imagination, which exalt wine as a mythical and fused element of the terroir.

\section{INDEX}

Mots-clés : Chapuzet (Jean-Charles), vin, polar, terroir

Keywords : Chapuzet (Jean-Charles), wine, thriller, terroir 
AUTEUR

JOSÉ DOMINGUES DE ALMEIDA

Un. Porto - APEF - ILC ML

jalmeida[at]letras.up.pt 